\title{
Relative Maturation of Peripheral and Central Regions of the Human Brainstem From Preterm to Term and the Influence of Preterm Birth
}

\author{
ZE D. JIANG, DOROTHEA M. BROSI, YUN Y. WU, AND ANDREW R. WILKINSON
}

Children's Hospital [Z.D.J., Y.Y.W.], Fudan University, Shanghai 200032, China; Neonatal Unit [Z.D.J., D.M.B., A.R.W.], Department of Paediatrics, University of Oxford, Oxford OX3 9DU, United Kingdom

\begin{abstract}
Relative maturation of peripheral and central regions of the neonatal brainstem was studied using brainstem auditory evoked responses in 174 healthy preterm infants (gestational age 30-36 wk). From 30- to 42-wk postconceptional age (PCA), I-III and III-V intervals shortened with increasing age. It was difficult to detect any apparent differences in maturational rate between the two intervals. However, III-V/I-III interval ratio decreased with increasing age, indicating that from preterm to term III-V interval shortens more than I-III interval. During term period (37- to 42-wk PCA), I-III interval was similar to term controls, but III-V interval was significantly longer and III-V/I-III interval ratio was significantly greater than controls at 37- to 38-wk PCA and 39-40 wk PCA and was the same as controls at 41-42 wk. Therefore, from 30- to 42-wk PCA maturation of central regions of the brainstem, reflected by III-V interval, is relatively faster than peripheral regions, reflected by I-III interval which seems to be already more mature than III-V interval before $30 \mathrm{wk}$. Maturation in central regions in preterm infants is relatively delayed at early term, but "catches-up" later, whereas peripheral regions already reach normal level of maturation at early term. Preterm birth slightly delays early maturation of central brainstem regions. (Pediatr Res 65: 657-662, 2009)
\end{abstract}

$\mathrm{D}$ uring the preterm period, there are dramatic developmental changes in the brainstem (1). Previous studies have shown that the immature brainstem can be damaged by various unfavorable perinatal conditions, e.g., hypoxia-ischemia (2). Recent studies revealed that brain structure may be abnormal in preterm infants and preterm birth is associated with regional cerebral tissue reductions $(3,4)$. It remains unclear whether the rate of maturation in the more peripheral and more central regions of the brainstem is equal or different during earlier life and what influence the preterm birth exerts on this maturation. The understanding will help improve the care and management of very young infants, particularly for those who may be at risk of developmental abnormalities.

In addition to detecting auditory abnormalities, brainstem auditory evoked responses (BAERs) have been used to study functional status and maturation of the, specifically auditory, brainstem (5-7). The responses, generating from specific brainstem auditory relay nuclei, are extremely complex and

Received August 19, 2008; accepted January 24, 2009.

Correspondence: Ze Dong Jiang, M.D., Ph.D., Neonatal Unit, Department of Paediatrics, University of Oxford, John Radcliffe Hospital, Headington, Oxford OX3 9DU, United Kingdom; e-mail: zedong.jiang@paediatrics.ox.ac.uk

Supported by WellChild and Medical School of Oxford University, United Kingdom. are under multiple inhibiting and facilitating influences from the rest of the CNS. Maturation of the BAER is associated with myelination of axons, formation of central synaptic connections, and axonal diameter and has been found to correlate with maturation of the brainstem, as well as the auditory pathway $(8-10)$. During early life, the BAER undergoes tremendous maturational changes, characterized by a shortening of wave latencies and an increase in wave amplitudes of the response (11-14). Furthermore, a shortening of the intervals between BAER wave peaks (i.e., interpeak intervals) with increasing age has been well documented.

In BAER wave form, wave I, III, and V, the three major and reliable waves, originate from sequentially activated neurons at successively higher levels of the brainstem auditory pathway $(6,15)$. Waves I and II originate exclusively from the eighth nerve, wave III is mainly generated by the neurons in the cochlear nucleus. Wave $\mathrm{V}$ is most likely originated by the lateral lemniscus as it enters the inferior colliculus, although the slow negative potential after the peak of wave $\mathrm{V}$ is likely to be a dendritic potential of the inferior colliculus. The interval between waves I and $\mathrm{V}$, i.e., $\mathrm{I}-\mathrm{V}$ interpeak interval, is the most commonly used BAER variable in the assessment of functional status and maturation of the brainstem. This interval comprised two subcomponents or smaller intervals, the earlier subcomponent I-III interval and the later one III-V interval. The I-III interval generally reflects functional status of the more peripheral regions of the auditory brainstem, whereas the III-V interval reflects functional status of the more central regions.

The human brain is known to develop in a caudal-to-rostral, or peripheral-to-central, fashion (16). The maturation of neuronal function in the more peripheral and more central regions of the brainstem may proceed at different rates at different stages of development. We hypothesize that the rate of maturation in the more peripheral and more central regions of the auditory brainstem is unequal, and the more peripheral regions mature earlier than the more central regions, and that preterm birth may affect the maturational rate. To test the hypotheses, we studied the changes in I-III and III-V intervals and, in particular, III-V/I-III interval ratio, which reflects the relative

Abbreviations: BAER, brainstem auditory evoked response; dB nHL, decibel normal hearing level; PCA, postconceptional age 
changes in the two intervals, during the preterm and term periods to detect any differences in the rate of maturation between the two intervals from preterm to term.

The BAER at term age in both preterm and term infants has long been studied. However, little information is available regarding changes in BAER at different stages during the term period [i.e., from 37- to 42-wk postconceptional age (PCA), defined as gestational age plus chronological age]. Thus, this study also examined changes in BAER intervals at different stages during the term period and compared the changes between healthy preterm and term infants to identify any differences.

\section{SUBJECTS AND METHODS}

Subjects. We recruited 174 healthy preterm infants, with a gestational age (GA) 30-36 (32.3 \pm 1.8$) \mathrm{wk}$ and birthweight 1255-3250 g (1859 $\pm 378 \mathrm{~g})$, between April 2003 and September 2007. Apgar scores were 8-10 at 1 and $5 \mathrm{~min}$. Part of the data obtained at term age from 26 of these infants had been presented before (17). All infants were judged to be stable in the nursery. None had any perinatal complications or major conditions. Any infant who had documented peripheral auditory impairment was excluded from study entry to avoid any influence of peripheral auditory impairment on measurements of BAER components. All subjects passed our neonatal hearing screening program with distortion product otoacoustic emission. Any infants with poor MLS BAER waveforms due to artifacts were also excluded.

A further group of 56 healthy term infants, with a GA 37-42 wk (39.1 \pm 1.2), was recruited as the term controls. The birthweight ranged between 2560

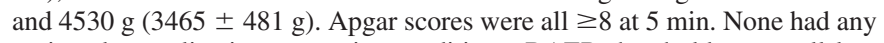
perinatal complications or major conditions. BAER thresholds were all less than $20 \mathrm{~dB}$ normal hearing level (nHL, referred to the average thresholds obtained from 20 adults tested under the identical conditions to those used for the babies) at the time of testing.

Study of BAER in the preterm infants during the preterm period and the term controls was carried out on d $2-\mathrm{d} 7$ after birth. Sixty-five, with a GA $30-35 \mathrm{wk}(32.3 \pm 2.1)$, of the 174 preterm infants were also studied during the term period (37- to 42-wk PCA). No statistically significant differences were found in the PCA between the preterm infants at term $(39.3 \pm 1.5 \mathrm{wk})$ and the term controls $(39.3 \pm 1.3 \mathrm{wk})$. Based on the PCA at which BAER recording was made, the preterm infants were divided into three age groups, including 30-32 $(n=55), 33-34(n=59)$, and 35-36 $(n=60)$ wk. Of these infants, 65 were tested again $37-42 \mathrm{wk}$.

To detect any differences in these intervals between the two groups of infants at difference stage of the term period, we further divided the term period into three subterm ages, including 37 - to 38 -wk PCA $(n=23$ preterm and 18 term), 39-40 wk $(n=22$ preterm and 20 term), and $41-42 \mathrm{wk}(n=$ 20 preterm and 18 term).

BAER recording and analysis. Ethical approval of the study protocols was obtained from the Central Oxford Research Ethics Committee. Informed consent was obtained from the parents of each infant. BAER was recorded in a quiet room, with the infants lying supine in the cot during the recording. A Bravo Portable Evoked Potential System (Nicolet Biomedical, Madison, WI) was used to record and analyze BAER.

The procedures of BAER recording and analysis were the same as previous described $(11,16)$. Briefly, three gold-plated disk electrodes were placed at the middle forehead $(+)$, the ipsilateral (left) earlobe $(-)$, and the contralateral (right) earlobe (ground). BAER recording commenced soon after the infant fell asleep naturally, usually after a feed. Rarefaction clicks of $100 \mu \mathrm{s}$, delivered to the left ear through a TDH 39 headphone, were presented in the order of 21/s, 51/s, and 91/s in the first run and in reverse order in the second run. All infants were tested at the intensity of 60 decibel normal hearing level ( $\mathrm{dB}$ nHL). In those preterm infants who had a threshold of $20-30 \mathrm{~dB}$ nHL the intensity was increased to $70 \mathrm{~dB} \mathrm{nHL}$ to elicit better waveforms. The averaged brain responses to the click stimuli were amplified and filtered at 100-3000 Hz. Two thousand forty-eight evoked brain responses were averaged for each trial. Duplicated recordings were made to document the reproducibility.

Measurements of BAER variables were blind to the medical history of each subject. Latencies of waves I, III, and V and interpeak intervals of I-V, I-III, and III-V were measured, and III-V/I-III interval ratio was then calculated $(11,16)$. The measurements of two replicated BAER recordings to each stimulus condition were averaged for analysis. All data were analyzed at $\geq 40 \mathrm{~dB}$ above the threshold of each infant $(11,18)$. The click intensity above BAER threshold was $48.8 \pm 8.1 \mathrm{~dB}$ in the preterm infants and $48.6 \pm 5.4 \mathrm{~dB}$ in the term controls, which did not differ significantly.

Analysis of variance was used to compare between the data at term in the preterm and those in the term controls at the same PCA. Correlation and regression analyses were performed between BAER variables and age in PCA. A one-sample $t$ test of the slopes of interval-PCA functions was performed for each BAER interval to determine whether the slopes of the linear functions were consistently different from zero.

\section{RESULTS}

The results described later were those obtained at $21 / \mathrm{s}$ clicks, unless otherwise specified (at 51/s and 91/s clicks).

General age-related changes in interpeak intervals between 30- and 42-wk PCA. From 30- to 42-wk PCA, I-V, I-III, and III-V intervals in the preterm infants shortened with increasing age (Figs. 1-3). The shortening was more rapidly during the preterm period (30- to 37-wk PCA) than during the term period (37- to 42-wk PCA). No apparent differences were found in the rate of age-related change between the I-III and III-V intervals. With increasing age, the III-V/I-III interval ratio tended to shorten during both the preterm and term periods (from 30- to 42-wk PCA, Fig. 4). The I-V, I-III, and III-V intervals and III-V/I-III interval ratio were all correlated significantly and negatively with the PCA $(r=-0.38$ to 0.68 , all $p<0.001$ ).

Analysis of regression showed that both intercept and slope of the interval-PCA function were the greatest for I-V interval, second for III-V interval, and the smallest for I-III interval (Table 1). As shown by the slope of interval-PCA function, from 30- to 42-wk PCA, the I-V, I-III, and III-V intervals shortened at the rates $0.091,0.035$, and $0.057 \mathrm{~ms} / \mathrm{wk}$, respectively, and the III-V/I-III interval ratio decreased at

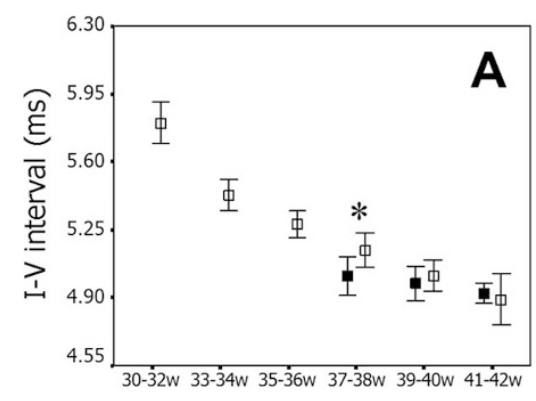

Age (PCA)

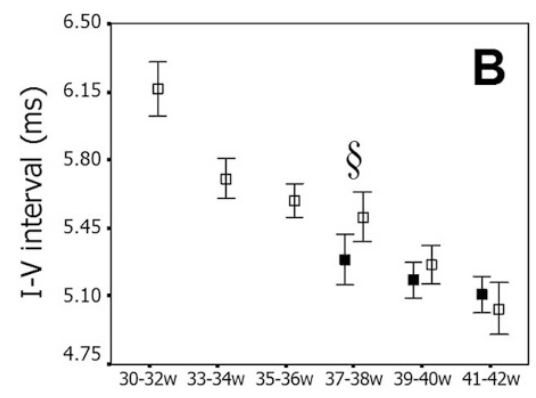

Age (PCA)

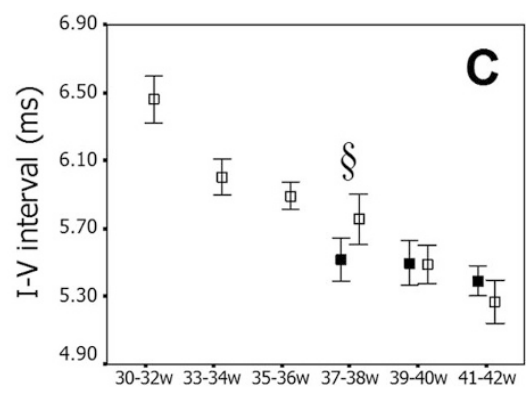

Age (PCA)

Figure 1. Mean and standard deviations of I-V interval at different PCA at 21/s (A), 51/s $(B)$, and 91/s $(C)$ clicks in preterm infants ( $\square$ ) and term controls ( $\square$ ). ${ }^{*} p<0.05, \S p<0.01$ for statistical comparison of I-V interval between preterm and term infants at the same PCA. 


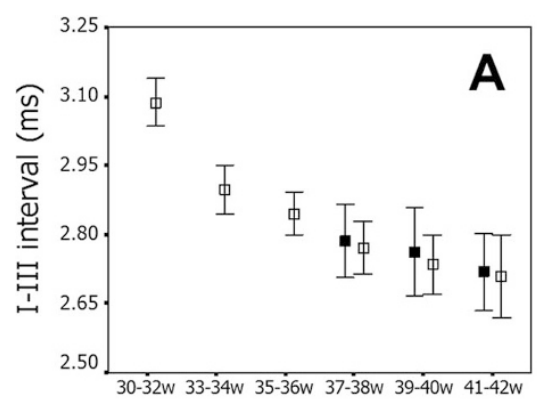

Age (PCA)

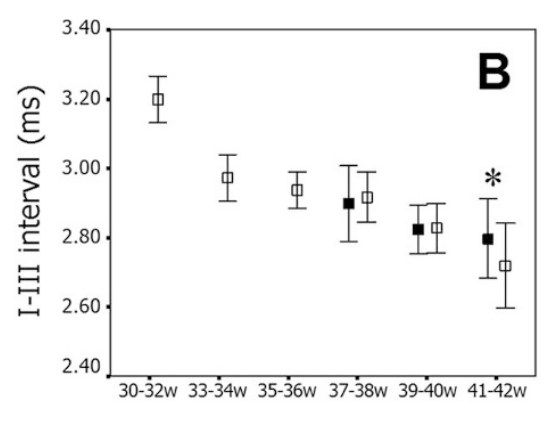

Age (PCA)

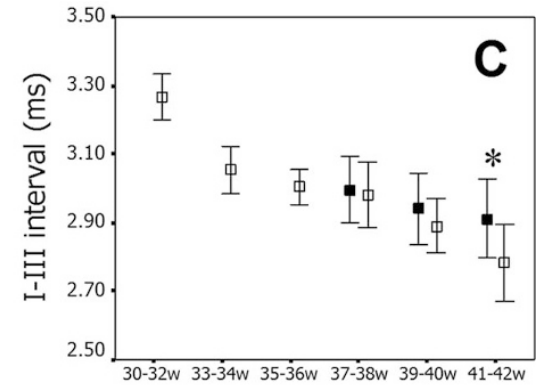

Age (PCA)

Figure 2. Mean and standard deviations of I-III interval at different PCA at 21/s (A), 51/s (B), and 91/s (C) clicks in preterm infants ( $\square$ ) and term controls ( $\square$ ). ${ }^{*} p<0.05$ for statistical comparison of I-III interval between preterm and term infants at the same PCA.

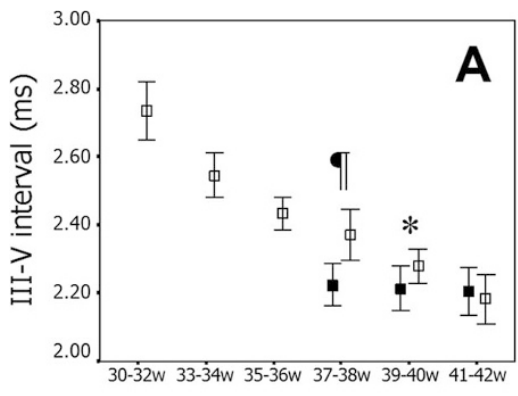

Age (PCA)
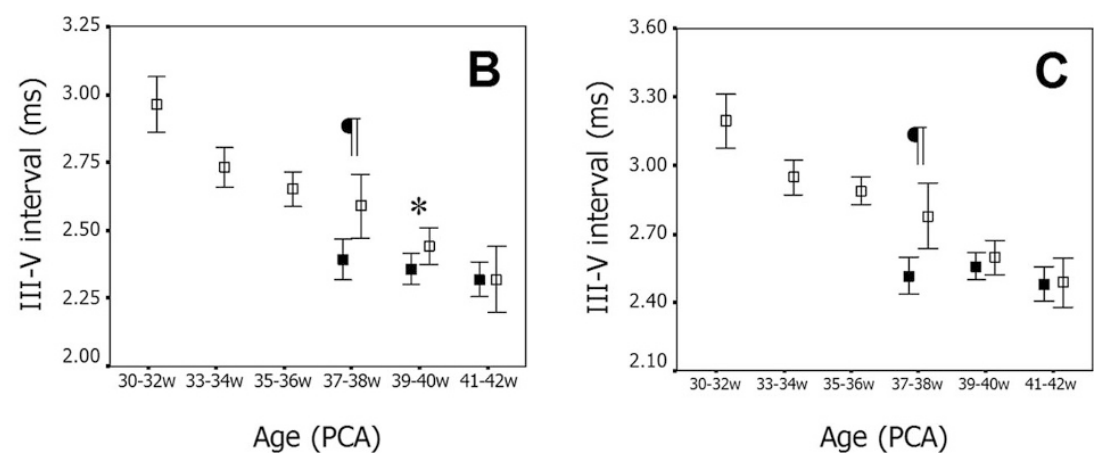

Figure 3. Mean and standard deviations of III-V interval at different PCA at 21/s $(A), 51 / \mathrm{s}(B)$, and 91/s $(C)$ clicks in preterm infants ( $\square$ ) and term controls (ם). ${ }^{*} p<0.05, \Phi p<0.001$ for statistical comparison of III-V interval between preterm and term infants at the same PCA.
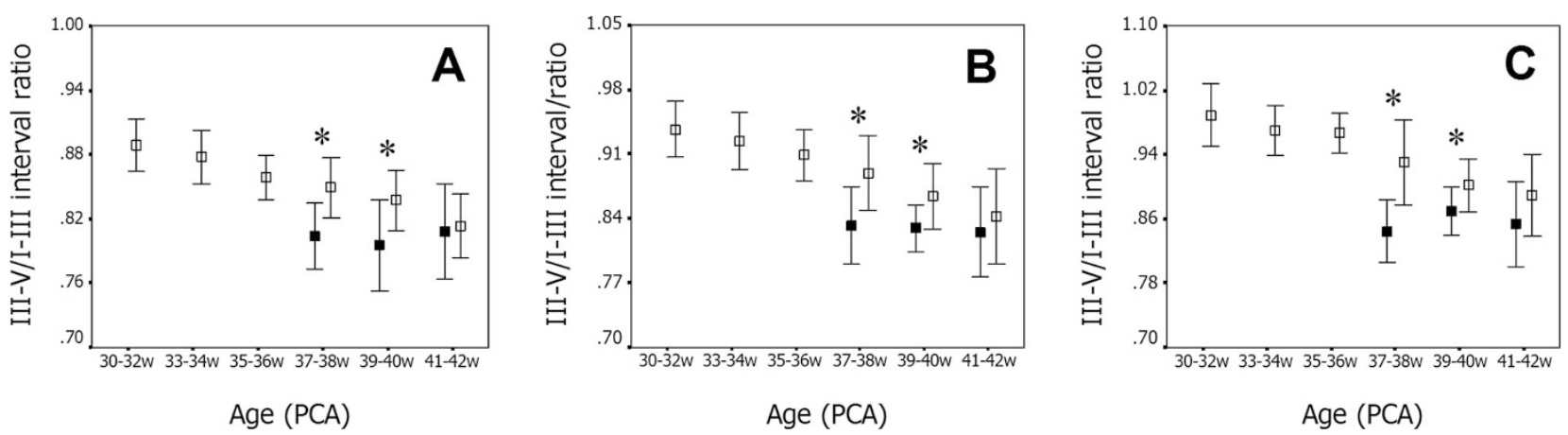

Figure 4. Mean and standard deviations of III-V/I-III interval ratio at different PCA at 21/s $(A), 51 / \mathrm{s}(B)$, and 91/s $(C)$ clicks in preterm infants ( $\square)$ and term controls $(\square) . * p<0.05$ for statistical comparison of III-V/I-III interval ratio between preterm and term infants at the same PCA.

0.008/wk. The slope of interval-PCA function was significantly greater for III-V than for I-III $(p<0.01)$.

To understand the age-related changes in BAER intervals at different developmental stages, we further examined the changes during the preterm and term periods separately.

Age-related changes in interpeak intervals during the preterm period. From 30- to 37-wk PCA, similar to the I-V interval (Fig. 1), both the I-III and III-V intervals in the preterm infants shortened rapidly, with the rapidest shortening occurring from 30- to 34-wk PCA (Figs. 2 and 3). These intervals were all correlated significantly and negatively with the PCA $(r=-0.47$ to 0.62 , all $p<0.001)$. Similarly, the III-V/I-III interval ratio decreased with increasing age (Fig. 4) and was correlated significantly and negatively with the PCA $(r=-0.20, p<0.01)$.
The intercept and slope of interval-PCA function were both the greatest for I-V, second for III-V and the smallest for I-III (Table 1). The slope of interval-PCA function indicated that I-V, I-III, and III-V intervals shortened at 0.127, 0.051, and $0.077 \mathrm{~ms} / \mathrm{wk}$, respectively. The III-V/I-III interval ratio decreased at a rate $0.009 / \mathrm{wk}$. The slope of the III-V intervalPCA function was significantly greater than that of the I-III interval-PCA function $(p<0.01)$.

Age-related changes in interpeak intervals during the term period. From 37- to 42-wk PCA, the interpeak intervals in the preterm infants continued to shorten with increasing age (Figs. 1-3), but the shortening was much smaller than during the preterm period. The III-V/I-III interval ratio also continued to decrease with increasing age (Fig. 4). The III-V and I-V intervals were correlated significantly and negatively with 
Table 1. Regression analysis of the relationship between BAER intervals at $21 / \mathrm{s}$ clicks and $\mathrm{PCA}$

\begin{tabular}{lrrrr}
\hline BAER variables & $\mathrm{I}-\mathrm{V}(\mathrm{ms})$ & $\mathrm{I}-\mathrm{III}(\mathrm{ms})$ & $\mathrm{III}-\mathrm{V}(\mathrm{ms})$ & $\mathrm{III}-\mathrm{V} / \mathrm{I}-\mathrm{III}$ \\
\hline Preterm at 30-42 wk & & & & \\
$a$ & 8.574 & 4.191 & 4.478 & 1.133 \\
$b$ & -0.091 & -0.035 & -0.057 & -0.008 \\
$r$ & -0.680 & -0.520 & -0.619 & -0.274 \\
$p$ & $<0.001$ & $<0.001$ & $<0.001$ & $<0.001$ \\
Preterm at 30-37 wk & & & & \\
$a$ & 9.757 & 4.709 & 5.220 & 1.189 \\
$b$ & -0.127 & -0.051 & -0.077 & -0.009 \\
$r$ & -0.615 & -0.467 & -0.545 & -0.203 \\
$p$ & $<0.001$ & $<0.001$ & $<0.001$ & $<0.010$ \\
Preterm at 37-42 wk & & & & \\
$a$ & 7.221 & 3.365 & 3.837 & 1.229 \\
$b$ & -0.056 & -0.016 & -0.039 & -0.010 \\
$r$ & -0.474 & -0.205 & -0.489 & -0.270 \\
$p$ & $<0.001$ & $>0.050$ & $<0.001$ & $<0.050$ \\
Term at 37-42 wk & & & & \\
$a$ & 6.175 & 3.386 & 2.770 & 0.865 \\
$b$ & -0.031 & -0.016 & -0.014 & -0.002 \\
$r$ & -0.281 & -0.142 & -0.166 & 0.030 \\
$p$ & $<0.050$ & $>0.050$ & $>0.050$ & $>0.050$ \\
\hline
\end{tabular}

$a$, regression intercept; $b$, regression slope; $r$, correlation coefficient; $p$, significance.

the PCA $(r=-0.49$ and -0.47 , both $p<0.001)$. However, the I-III interval, though also tended to shorten with increasing age, was not correlated significantly with the PCA. The III-V/IIII interval ratio was correlated weakly, but statistically significantly, and negatively with the PCA $(r=-0.27, p<0.05)$.

As indicated by the slope of interval-PCA function in Table 1, from 37- to 42-wk PCA, the I-V and III-V intervals shortened at a rate of 0.056 and $0.039 \mathrm{~ms} / \mathrm{wk}$, whereas the I-III interval did not change significantly with varying age. The III-V/I-III interval ratio decreased at a rate of $0.01 / \mathrm{wk}$.

Comparison of interpeak intervals during the term period between preterm and term infants. From 37- to 42-wk PCA, the $\mathrm{I}-\mathrm{V}$ interval in the term controls shortened slightly, whereas the I-III and III-V intervals did not show any significantly age-related changes. However, in the preterm infants, the three intervals all shortened with increasing age. The III-V/I-III interval ratio did not show any clear age-related changes in the term controls, but decreased with increasing age in the preterm infants.

In the term controls, the I-V interval was correlated significantly and negatively with the PCA $(r=-0.28, p<0.05)$, but neither the I-III interval nor the III-V interval had a significant correlation with the PCA. The III-V/I-III interval ratio was not correlated with the PCA, either. However, in the preterm infants, both the I-V and III-V intervals were correlated significantly and negatively with the PCA during the term period. This was also the case of the III-V/I-III interval ratio. Only I-III interval was not correlated with the PCA.

Regression analysis revealed that in the term controls the I-III and III-V intervals and III-V/I-III interval ratio did not change systematically with varying age, except the I-V interval, which shortened at $0.031 \mathrm{~ms} / \mathrm{wk}$ (Table 1 ). However, in the preterm infants, with increasing age the I-V and III-V intervals shortened and the III-V/I-III interval ratio decreased (Table 1). Only the I-III interval did not change systematically with varying age, which was similar to the term controls (Table 1). The slope of the I-V interval-PCA function was significantly steeper in the preterm infants than in the term controls $(p<0.01)$.

The measurements of the I-V, I-III, and III-V intervals in the preterm infants were $4.99 \pm 0.21,2.73 \pm 0.16$, and $2.26 \pm$ 0.13 , respectively. None differed significantly from those in the term controls $(4.97 \pm 0.15,2.76 \pm 0.15$, and $2.21 \pm 0.12$, respectively, for the three intervals). The III-V/I-III interval ratio $(0.83 \pm 0.07)$ was greater than in the term controls $(0.80 \pm 0.07, p<0.05)$.

We further compared the intervals between the preterm and term controls at each of the three subterm ages. The I-V interval in the preterm infants was significantly longer than in the term controls at 37- to 38-wk PCA $(p<0.05)$, slightly longer at 39-40 wk, and approached the term value at 41- to 42-wk PCA. The I-III interval at all 37-38, 39-40, and 41-42 wk PCA was similar to those in the term controls (Fig. $2)$. The III-V interval was significantly longer than in the term controls at 37- to 38-wk PCA $(p<0.001)$ remained longer, though less significantly, at 39- to 40-wk PCA $(p<0.05)$, and reached the term value at 41- to 42-wk PCA (Fig. 3). Similarly, the III-V/I-III interval ratio was greater than in the term controls at 37-38 and 39-40 wk ( $p<0.05$ and 0.05$)$, which was mainly due to the longer III-V interval in the preterm infants and almost the same as the term controls at 41- to 42-wk PCA.

Age-related changes at higher rates of click stimuli. At the click rates 51/s and 91/s, the age-related changes in the I-V, I-III, and III-V intervals and the III-V/I-III interval ratio in the preterm infants were generally similar to those obtained at 21/s clicks, with only slight differences (Figs. 1-4). No apparent differences were seen in the age-related changes between the I-III and III-V interval (Figs. 2 and 3). The III-V/I-III interval ratio tended to decrease with increasing age during both the preterm and term periods (Fig. 4). The results of correlation and regression analyses between BAER variables and PCA at 51/s and 91/s were also essentially similar to those at $21 / \mathrm{s}$ (Tables 2 and 3).

\section{DISCUSSION}

Age-related relative changes in BAER intervals from preterm to term and the value of III-V/I-III interval ratio in assessing the relative changes. From preterm to term, the I-III and III-V intervals shortened somewhat similarly with increasing age. As previously reported, based on the absolute values it is difficult to accurately compare maturational changes in the two intervals and detect any apparent differences in their rates of maturation (13). However, the agerelated change in the III-V/I-III interval ratio has made the differences apparent. The decrease in the interval ratio with increasing age implies that from preterm to term the agerelated change in the III-V interval is more than that in the I-III interval.

At term, as a single age group (37- to 42-wk PCA) the I-III and III-V intervals in the preterm infants did not differ significantly from those in the term infants. However, the 
Table 2. Regression analysis of the relationship between BAER intervals at $51 / \mathrm{s}$ clicks and $P C A$

\begin{tabular}{lrrrr}
\hline BAER variables & $\mathrm{I}-\mathrm{V}(\mathrm{ms})$ & $\mathrm{I}-\mathrm{III}(\mathrm{ms})$ & $\mathrm{III}-\mathrm{V}(\mathrm{ms})$ & $\mathrm{III}-\mathrm{V} / \mathrm{I}-\mathrm{III}$ \\
\hline Preterm at 30-42 wk & & & & \\
$a$ & 9.327 & 4.110 & 4.908 & 1.216 \\
$b$ & -0.104 & -0.041 & -0.063 & -0.009 \\
$r$ & -0.712 & -0.554 & -0.651 & -0.292 \\
$p$ & $<0.001$ & $<0.001$ & $<0.001$ & $<0.001$ \\
Preterm at 30-37 wk & & & & \\
$a$ & 10.321 & 4.794 & 5.534 & 1.288 \\
$b$ & -0.134 & -0.052 & -0.082 & -0.011 \\
$r$ & -0.610 & -0.452 & -0.551 & -0.226 \\
$p$ & $<0.001$ & $<0.001$ & $<0.001$ & $<0.010$ \\
Preterm at 37-42 wk & & & & \\
$a$ & 8.745 & 4.380 & 4.370 & 1.050 \\
$b$ & -0.088 & -0.039 & -0.049 & -0.005 \\
$r$ & -0.664 & -0.496 & -0.496 & -0.110 \\
$p$ & $<0.001$ & $<0.010$ & $<0.001$ & $>0.050$ \\
Term at 37-42 wk & & & & \\
$a$ & 7.158 & 3.762 & 3.297 & 1.004 \\
$b$ & -0.050 & -0.026 & -0.024 & -0.004 \\
$r$ & -0.346 & -0.184 & -0.282 & 0.092 \\
$p$ & $<0.010$ & $>0.050$ & $>0.050$ & $>0.050$ \\
\hline
\end{tabular}

$a$, regression intercept; $b$, regression slope; $r$, correlation coefficient; $p$ significance.

Table 3. Regression analysis of the relationship between BAER intervals at $91 /$ s clicks and PCA

\begin{tabular}{lrrrr}
\hline \multicolumn{1}{c}{ BAER variables } & $\mathrm{I}-\mathrm{V}(\mathrm{ms})$ & $\mathrm{I}-\mathrm{III}(\mathrm{ms})$ & $\mathrm{III}-\mathrm{V}(\mathrm{ms})$ & $\mathrm{III}-\mathrm{V} / \mathrm{I}-\mathrm{III}$ \\
\hline Preterm at 30-42 wk & & & & \\
$a$ & 9.842 & 4.502 & 5.339 & 1.306 \\
$b$ & -0.111 & -0.041 & -0.069 & -0.010 \\
$r$ & -0.730 & -0.557 & -0.661 & -0.308 \\
$p$ & $<0.001$ & $<0.001$ & $<0.001$ & $<0.001$ \\
Preterm at 30-37 wk & & & & \\
$a$ & 10.714 & 4.886 & 5.830 & 1.321 \\
$b$ & -0.137 & -0.053 & -0.084 & -0.010 \\
$r$ & -0.617 & -0.456 & -0.530 & -0.196 \\
$p$ & $<0.001$ & $<0.001$ & $<0.001$ & $<0.050$ \\
Preterm at 37-42 wk & & & & \\
$a$ & 8.938 & 4.336 & 4.600 & 1.135 \\
$b$ & -0.087 & -0.037 & -0.050 & -0.006 \\
$r$ & -0.591 & -0.416 & -0.454 & -0.125 \\
$p$ & $<0.001$ & $<0.010$ & $<0.001$ & $>0.050$ \\
Term at 37-42 wk & & & & \\
$a$ & 6.694 & 3.831 & 2.898 & 0.743 \\
$b$ & -0.031 & -0.022 & -0.010 & -0.003 \\
$r$ & -0.203 & -0.167 & -0.103 & 0.056 \\
$p$ & $>0.050$ & $>0.050$ & $>0.050$ & $>0.050$ \\
\hline
\end{tabular}

$a$, regression intercept; $b$, regression slope; $r$, correlation coefficient; $p$ significance.

III-V/I-III interval ratio was significantly greater than in the term infants, suggesting that there are differences in the I-III and III-V intervals between preterm and term infants. The differences are too small to be shown by the absolute values of the two intervals. Therefore, the III-V/I-III interval ratio can detect some differences or abnormalities that cannot be shown by analyzing absolute interval values only.

Despite some slight differences between different rates of clicks, the age-related changes in III-V/I-III interval ratio were basically similar at all rates $21 / \mathrm{s}, 51 / \mathrm{s}$, and $91 / \mathrm{s}$. Therefore, from preterm to term the relative maturational changes in the I-III and III-V intervals are essentially independent of changing repetition rate of clicks.

Relative maturation of the peripheral and central regions of the brainstem from preterm to term. The possible interpretation of the age-related change in the III-V/I-III interval ratio in preterm infants relies on the assumption of the sites of generation of waves I, III, and V. As often expressed in the literature, the generators of BAER waves are located at moreor-less discrete locations in the auditory nerve (wave I), the more peripheral (cochlear nuclei, wave III) and more central regions of the brainstem (lateral lemniscus and inferior colliculus, wave V) $(6,15)$. Neural function in the more peripheral regions of the brainstem is reflected by the I-III interval, whereas the function in the more central regions is reflected by the III-V interval.

The III-V/I-III interval ratio in our preterm infants showed clear age-related changes, suggesting that the rates of maturation in the more peripheral and more central regions of the auditory brainstem are unequal. The decrease in the interval ratio with increasing age implies that age-related change is more significant in the III-V interval than in the I-III interval. It seems that maturation of the more central (or rostral) regions of the brainstem is relatively faster than the more peripheral (or caudal) regions. These findings seem to contradict to the caudal-to-rostral, or peripheral-to-central, fashion of brain development.

Nevertheless, this relatively faster maturation in the more central regions of the brainstem does not necessarily simply imply that the more central regions are more mature than the more peripheral regions during this period studied. It is more likely that before or around 30-wk PCA, the more peripheral regions are already relatively more mature than the more central regions. After 30-wk PCA, further maturation in the more peripheral regions is getting slower, whereas in the more central regions, which is less mature, further maturation becomes relatively faster, as reflected by the decrease in III-V/ I-III interval ratio with increasing age. This process continues up to the end of the term period.

At 37- to 38-wk PCA, the I-III interval in the preterm infants already reached the term value while the III-V interval remained significantly longer than term value. Apparently, even though during the preterm period (30- to 37-wk PCA) maturation in the more central regions of the brainstem is faster than the more peripheral regions, by early term age the more central regions remain relatively less mature than the more peripheral regions.

Taken together, our findings are consistent with our hypothesis that the rate of maturation in the more peripheral and more central regions of the brainstem is unequal and the more peripheral regions mature earlier than the more central regions. This still follows the principle of caudal-to-rostral, or peripheral-to-central, fashion of brain development.

Influence of preterm birth on relative maturation of the peripheral and central regions of the brainstem. Preterm infants are known to be at risk of neurodevelopmental abnormalities (19-21). Preterm birth may also affect maturation of evoked potentials, including BAER, although there remain controversies $(11,12,17,22-24)$. This study found that the 
III-V interval in preterm infants is less mature than term infants from 37- to 40-wk PCA, but reaches term value at 41-42 wk. This implies that in preterm infants there is delay in maturation in the more central regions of the brainstem at earlier term age, but this delay "catches-up" at later term age. In contrast, the I-III interval in preterm infants already reaches normal level of maturation at early term age. Apparently, preterm birth and associated perinatal conditions result in a slight maturational delay in the more central regions of brainstem from preterm up to earlier term periods. This is generally consistent with our second hypothesis that preterm birth affects the rate of maturation of the brainstem. Nevertheless, the more peripheral regions are not significantly affected by preterm birth.

Similar to previous findings $(7,11,12,17)$, when our data obtained between 37- and 42-wk PCA were pooled together as a single age group, there was no significant difference in the $\mathrm{I}-\mathrm{V}$ interval between the preterm and term infants. However, when the term period was divided into three subterm ages, differences appeared between the preterm and term infants. The I-V interval in the preterm infants was significantly longer than in the term infants at the earliest term age (37- to 38-wk PCA), although the difference disappeared later (39- to 42-wk PCA). The III-V intervals were significantly longer than in the term infants at the earliest and mid term ages (37to 40-wk PCA), and reached the term value at the latest term age (41- to 42-wk PCA). In contrast, the I-III interval was similar to that in the term infants throughout the term period (37- to 42-wk PCA).

In conclusion, this study revealed some differences in the rate of maturation between I-III and III-V intervals during the preterm and term periods. In preterm infants, BAER reaches term value at 37- to 38-wk PCA for I-III interval, 39- to 40-wk PCA for I-V interval, and 41- to 42-wk PCA for III-V interval. The III-V/I-III interval ratio helps detect and localize abnormalities in the more peripheral or more central regions of the brainstem.

Our results suggest that from 30- to 42-wk PCA maturation in the more central regions of the brainstem is faster than the more peripheral regions whereas the more peripheral regions is more mature than the more central regions before or around 30-wk PCA. Preterm birth slightly delays early maturation of the more central regions of the brainstem, with no appreciable effect on the more peripheral regions. The delayed maturation "catches up" and reaches the maturational status in term infants by later term age.

\section{REFERENCES}

1. Darnall RA, Ariagno RL, Kinney HC 2006 The late preterm infant and the control of breathing, sleep, and brainstem development: a review. Clin Perinatol 33:883-914

2. Buller KM, Wixey JA, Pathipati P, Carty M, Colditz PB, Williams CE, Scheepens A 2008 Selective losses of brainstem catecholamine neurons after hypoxia-ischemia in the immature rat pup. Pediatr Res 63:364-369

3. Inder TE, Warfield SK, Wang H, Hüppi PS, Volpe JJ 2005 Abnormal cerebral structure is present at term in premature infants. Pediatrics 115:286-294

4. Thompson DK, Warfield SK, Carlin JB, Pavlovic M, Wang HX, Bear M, Kean MJ, Doyle LW, Egan GF, Inder TE 2007 Perinatal risk factors altering regional brain structure in the preterm infant. Brain 130:667-677

5. Volpe JJ 2001 Specialized studies in the neurological evaluation. In: Volpe JJ (ed) Neurology of the Newborn, 4th ed. Philadelphia, WB Saunders, pp 134-177

6. Wilkinson AR, Jiang ZD 2006 Brainstem auditory evoked response in neonatal neurology. Semin Fetal Neonatal Med 11:444-451

7. Eggermont JJ, Salamy A 1988 Maturational time course for the ABR in preterm and full term infants. Hear Res 33:35-48

8. Moore JK, Perazzo LM, Braun A 1995 Time course of axonal myelination in the human brainstem auditory pathway. Hear Res 87:21-31

9. Moore JK, Guan YL, Shi SR 1997 Axogenesis in the human fetal auditory system, demonstrated by neurofilament immunohistochemistry. Anat Embryol (Berl) 195:15-30

10. Ponton CW, Moore JK, Eggermont JJ 1996 Auditory brain stem response generation by parallel pathways: differential maturation of axonal conduction time and synaptic transmission. Ear Hear 17:402-410

11. Jiang ZD, Brosi DM, Wilkinson AR 2002 Auditory neural responses to click stimuli of different rates in the brainstem of very preterm babies at term. Pediatr Res $51: 454-459$

12. Jiang ZD 1995 Maturation of the auditory brainstem in low risk preterm infants: a comparison with age-matched full term infants up to 6 years. Early Hum Dev 42:49-65

13. Krumholz A, Felix JK, Goldstein PJ, McKenzie E 1985 Maturation of the brain stem auditory evoked potential in premature infants. Electroencephalogr Clin Neurophysiol 62:124-134

14. Rotteveel JJ, de Graaf R, Colon EJ, Stegeman DF, Visco YM 1987 The maturation of the central auditory conduction in preterm infants until three months post term. II. The auditory brainstem responses (ABRs). Hear Res 26:21-35

15. Moore JK 1987 The human auditory brain stem as a generator of auditory evoked potentials. Hear Res 29:33-43

16. Lagercrantz H, Srinivasan M 1991 Development and function of neurotransmitter/ modulator systems in the brain stem. In: Hanson MA (ed) The Fetal and Neonatal Brain Stem: Developmental and Clinical Issues. Cambridge, Cambridge University Press, pp 1-20

17. Jiang ZD, Wilkinson AR 2008 Normal brainstem responses in moderately preterm infants. Acta Paediatr 97:1366-1369

18. Jiang ZD, Yin R, Shao XM, Wilkinson AR 2004 Brain-stem auditory impairment during the neonatal period in term infants after asphyxia: dynamic changes in brain-stem auditory evoked response to clicks of different rates. Clin Neurophysiol 115:1605-1615

19. Morris BH, Smith KE, Swank PR, Denson SE, Landry SH 2002 Patterns of physical and neurologic development in preterm children. J Perinatol 22:31-36

20. Olsén P, Vainionpää L, Pääkkö E, Korkman M, Pyhtinen J, Järvelin MR 1998 Psychological findings in preterm children related to neurologic status and magnetic resonance imaging. Pediatrics 102:329-336

21. Pietz J, Peter J, Graf R, Rauterberg-Ruland I, Rupp A, Sontheimer D, Linderkamp O 2004 Physical growth and neurodevelopmental outcome of nonhandicapped low-risk children born preterm. Early Hum Dev 79:131-143

22. Pasman JW, Rotteveel JJ, de Graaf R, Stegeman DF, Visco YM 1992 The effect of preterm birth on brainstem, middle latency and cortical auditory evoked responses (BMC AERs). Early Hum Dev 31:113-129

23. Pasman JW, Rotteveel JJ, de Graaf R, Maassen B, Visco YM 1996 The effects of early and late preterm birth on brainstem and middle-latency auditory evoked responses in children with normal neurodevelopment. J Clin Neurophysiol 13:234241

24. Mikkola K, Kushnerenko E, Partanen E, Serenius-Sirve S, Leipälä J, Huotilainen M, Fellman V 2007 Auditory event-related potentials and cognitive function of preterm children at five years of age. Clin Neurophysiol 118:1494-1502 